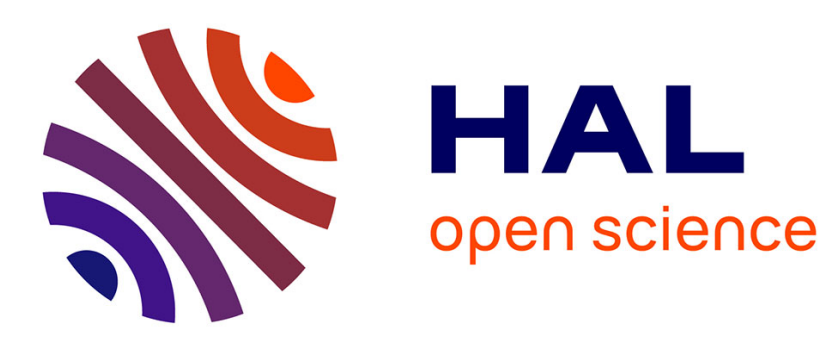

\title{
Convergence of iterative schemes for multivalued quasi-variational inclusions
}

Abdellatif Moudafi, Muhammad Aslam Noor

\section{To cite this version:}

Abdellatif Moudafi, Muhammad Aslam Noor. Convergence of iterative schemes for multivalued quasivariational inclusions. Positivity, 2004, 8 (1), pp.75-84. 10.1023/B:POST.0000023199.34934.9f . hal00780762

\section{HAL Id: hal-00780762 \\ https://hal.univ-antilles.fr/hal-00780762}

Submitted on 24 Jan 2013

HAL is a multi-disciplinary open access archive for the deposit and dissemination of scientific research documents, whether they are published or not. The documents may come from teaching and research institutions in France or abroad, or from public or private research centers.
L'archive ouverte pluridisciplinaire HAL, est destinée au dépôt et à la diffusion de documents scientifiques de niveau recherche, publiés ou non, émanant des établissements d'enseignement et de recherche français ou étrangers, des laboratoires publics ou privés. 


\title{
Convergence of Iterative Schemes for Multivalued Quasi-Variational Inclusions
}

\author{
Abdellatif Moudafi* and Muhammad Aslam Noor ${ }^{* *}$
}

\begin{abstract}
Relying on the resolvent operator method and using Nadler's theorem, we suggest and analyze a class of iterative schemes for solving multivalued quasi-variational inclusions. In fact, by considering problems involving composition of mutivalued operators and by replacing the usual compactness condition by a weaker one, our result can be considered as an improvement and a significant extension of previously known results in this field.
\end{abstract}

2000 AMS Subject Classification: 49J40, 90C33.

Keywords: Variational inclusions, convergence, monotone operators, pseudo-Lipschitz conditions.

\section{Introduction}

Quasi-variational inclusions are being used as mathematical programming models to study a large number of equilibrium problems arising in finance, economics, transportation, optimization, operations research and engineering sciences, see, for example [3, 6, 7] and the references therein. They have been extended and generalized in different directions by using novel and innovative techniques and ideas, both for their own sake and for their applications. In recent years, much attention has been given to develop efficient and implementable numerical methods including the projection method and its variant forms, Wiener-Hopf (normal) equations, linear approximation, auxiliary principle, proximal-point algorithm and descent framework for solving variational inequalities and related optimization problems. It is well known that the projection methods and its variant forms; and Wiener-Hopf equation techniques cannot be used to suggest and analyze iterative methods for solving quasi-variational inequalities due to the presence of the nonlinear term. This fact motivated to develop another technique, which involves the use of the resolvent operator associated with maximal monotone operator. Using this technique, one shows that the variational inclusions are equivalent to a fixed point problem. This alternative formulation was used to develop numerical methods for solving various classes of variational inclusions and related problems, see [17, 18]. The origin of this technique can be traced back to Martinet [12] and Rockafellar [22]. The major difficulty with these methods is that the operator $I+\lambda A$ may be hard to invert. One alternative of the previous difficulty is to decompose the given operator into the sum of two maximal monotone operators, whose resolvent are easier to evaluate than the resolvent 
of the original operator. Such a method is known as the operator splitting method. This can lead to the development of very efficient methods, since one can treat each part of the original operator independently. The operator splitting methods and related techniques have been analyzed and studied by many authors including Passty [19], Glowinski and le Tallec [10], and Tseng [24]. For an excellent account of the alternating direction implicit (splitting) methods, see Ames [1]. It is worth mentioning that if the nonlinear term involving the variational inequalities is the indicator function of a closed convex set in a Hilbert space, then the resolvent operator is equal to the projection operator. Consequently the resolvent equations are equivalent to the Wiener-Hopf (normal) equations, which were introduced by Shi [23] and Robinson [21] in relation with the classical variational inequalities.

The aim of this paper is twofold. First, is to suggest and analyze a new class of iterative schemes for solving the composite quasi-variational inclusions using the resolvent equation technique. The second is to prove a convergence result without the usual compactness condition. Our result includes the Ishikawa, Mann and Noor iterations for solving variational inclusions (inequalities) as special cases. We also study the convergence criteria of these new methods. Our result extends and generalizes the previously known results.

\section{Preliminaries}

Let $\mathcal{H}$ be a real Hilbert space whose inner product and norm are denoted by $\langle\cdot, \cdot\rangle$ and $|\cdot|$ respectively. Let $C B(\mathcal{H})$ be a familly of all nonempty bounded closed subsets of $\mathcal{H}$. Let $T, V: \mathcal{H} \rightarrow C B(\mathcal{H}), N(. ..) \rightarrow 2^{\mathcal{H}}$ be multivalued operators, $g: \mathcal{H} \rightarrow \mathcal{H}$ be a single-valued operator and $A(.,):. \mathcal{H} \times \mathcal{H} \rightarrow \mathcal{H}$ be a nonlinear one.

We consider the problem of finding $u \in \mathcal{H}, w \in T(u), y \in V(u)$ such that

$$
0 \in N(w, y)+A(g(u), u)
$$

Inclusion of type (2.1) is called multivalued quasi-variational inclusion, which has many important and useful applications in pure and applied sciences. Indeed, a number of problems arising in structural analysis, mechanics, composite problems, and economics can be studied in the framework of multivalued quasi-variational inclusions, see, for example [6,7]. For special choices of the operators, one can recover a large number of complementarity and quasi-complementarity problems. We would like to mention that the problem for finding a zero of the sum of two maximal monotone operators, location problem, finding a minimizer of the sum of two convex functions, various classes of variational inequalities are special cases of problem (2.1). We will give a special attention to the interesting case when $A_{u}$ is the normal cone of a nonempty closed convex set $K(u)$ (or equivalently $A_{u}$ is the partial differential operator of the indicator function of $K(u))$. In this case $(2.1)$ reduces to the following problem

Find $u \in \mathcal{H}, w \in T(u), y \in V(u), g(u) \in K(u)$ such that

$$
\langle N(w, y), v-g(u)\rangle \geq 0, \quad \text { for all } \quad v \in K(u),
$$


which in turn reduces to the following multivalued implicit complementarity problem

$$
g(u) \in K(u), N(w, y) \in K^{*}(u) \text {, and }\langle N(w, y), g(u)\rangle=0,
$$

where $K^{*}(u)=\{u \in \mathcal{H},\langle u, v\rangle \geq 0, \quad \forall v \in K(u)\}$ stands for the polar cone to $K(u)$ and $N(w, y)$ denotes, throughout this paper, an element of the set $N(w, y)$.

Now, let us recall that if $T$ is a maximal monotone operator, then the resolvent operator, $J_{\lambda}^{T}$, associated with $T$ is defined by

$$
J_{\lambda}^{T}(u)=(I+\lambda T)^{-1}(u), \quad \text { for all } \quad u \in H,
$$

where $\lambda>0$ is a constant and $I$ is the identity operator. The resolvent is a single-valued operator which is nonexpansive. Namely, $\left|J_{\lambda}^{T}(x)-J_{\lambda}^{T}(y)\right| \leq|x-y| \forall x, y \in \mathcal{H}$.

We also recall that for all $x \in \operatorname{dom} T:=\{x \in \mathcal{H}, T(x) \neq \emptyset\}$, the set $T(x)$ is closed and convex. Finally, we recall that the range of $T$ is given by $\mathrm{R}(T)=\{y \in \mathcal{H}, \exists x \in \mathrm{d}$ om $T ; y \in$ $T(x)\}$, that the composition of multi-valued operators is defined as $A B(x):=\{z \in \mathcal{H}, \exists y \in$ $B(x)$ with $z \in A y\}$ and that the Hausdorff distance between $C$ and $D$ is defined by

$$
\mathcal{H a u s}(C, D)=\max \{\mathrm{e}(C, D), \mathrm{e}(D, C)\}
$$

where the excess of the set $C \in C B(\mathcal{H})$ on a set $D \in C B(\mathcal{H})$ is defined as

$$
\mathrm{e}(C, D)=\sup _{x \in C} \inf _{y \in D}|x-y|
$$

Since the operator $A(.,$.$) is a maximal monotone, it is maximal monotone with respect to$ the first argument, so we set $A_{y}(\cdot):=A(\cdot, y)$.

Definition. For all $u_{1}, u_{2} \in \mathcal{H}$, the operator $N$ is said to be

(a) strongly monotone with respect to the first argument and with respect to $T$, if there exists a constant $\alpha$ such that

$$
\left\langle N_{y}\left(w_{1}\right),-N_{y}\left(w_{1}\right),, u_{1}-u_{2}\right\rangle \geq \alpha\left|u_{1}-u_{2}\right|^{2}, \quad \forall y \in \mathcal{H}, w_{i} \in T u_{i}, i=1,2 .
$$

(b) Lipschitz continuous with respect to the first argument, if there exists a constant $\beta>0$ such that

$$
\mathcal{H a u s}\left(N_{y}\left(w_{1}\right), N_{y}\left(w_{2}\right)\right) \leq \beta\left|w_{1}-w_{2}\right|, \quad \forall y \in \mathcal{H}, w_{i} \in \mathrm{R}(T), i=1,2,
$$

which is well defined because $\mathrm{R}(T)$ is bounded.

(c) Lipschitz continuous with respect to the second argument, if there exists a constant $\gamma>0$ such that

$$
\left.\mid N\left(w, y_{1}\right)-N\left(w, y_{2}\right)\right)|\leq \gamma| y_{1}-y_{2} \mid, \quad \forall w \in \mathcal{H}, y_{i} \in \mathcal{H}, i=1,2
$$

Now let us state a condition on the resolvent operator and a result by Nadler which will be needed in the sequel.

We assume that, for all $u, v, w \in \mathcal{H}$, the resolvent operator $J_{\lambda}^{A_{u}}(\cdot)$ satisfies the condition

$$
\left|J_{\lambda}^{A_{u}}(w)-J_{\lambda}^{A_{v}}(w)\right| \leq \nu|u-v|,
$$


where $\nu>0$ is a constant.

This condition is satisfied when the operator $A$ is monotone jointly with respect to the two arguments. Since it is better to have a condition on the operator (i.e. the data) than on its resolvent, we will establish here that (2.2) is also verified under a Lipschtiz condition on restrictions of the graph of the operator $A_{y}$ on closed balls centred at the origin.

Lemma: (Nadler [16]). Let $E$ be a complete metric space, $T: E \rightarrow C B(E)$ be a multivalued operator. Then for any $\varepsilon>0$ and for any given $x, y \in E, u \in T(x)$, there exists $v \in T(y)$ such that

$$
\operatorname{dist}(u, v) \leq(1+\varepsilon) \mathcal{H} \text { a } u s(T x, T y),
$$

where $\mathcal{H a u s}(\cdot, \cdot)$ is the Hausdorff metric on $C B(E)$.

\section{The main result}

To begin with, let us give an alternative fixed point formulation of the problem (2.1) which is very useful from both theoretical and numerical analysis points of view. We will use it to propose some iterative schemes for solving the considered problem. Indeed, let $u$ be a solution of (2.1), then for any $\lambda>0$, we can write

$$
0 \in \lambda N(w, y)+\lambda A(g(u), u) .
$$

In other words

$$
g(u)-\lambda N(w, y) \in\left(I+\lambda A_{u}\right)(g(u))
$$

thus

$$
g(u)=J_{\lambda}^{A_{u}}(g(u)-\lambda N(w, y)) .
$$

The latter relation can be rewritten as

$$
u=u-g(u)+J_{\lambda}^{A_{u}}(g(u)-\lambda N(w, y)),
$$

where $N(w, y)$ stands for an element of the set $N(w, y)$.

Frow (2.3) and Nadler's lemma, we derive the following unified iterative scheme.

Algorithm. For a given $u_{0} \in \mathcal{H}$, compute the sequences $\left\{u_{n}\right\},\left\{z_{n}\right\}$ by the iterative rules:

$$
\left\{\begin{array}{r}
z_{n}=\left(1-\beta_{n}\right) u_{n}+\beta_{n}\left(u_{n}-g\left(u_{n}\right)+J_{\lambda}^{A_{u_{n}}}\left(g\left(u_{n}\right)-\lambda N\left(w_{n}, y_{n}\right)\right)\right. \\
u_{n+1}=\left(1-\alpha_{n}\right) u_{n}+\alpha_{n}\left(z_{n}-g\left(z_{n}\right)+J_{\lambda}^{A_{z_{n}}}\left(g\left(z_{n}\right)-\lambda N\left(\tilde{w}_{n}, \tilde{y}_{n}\right)\right)\right.
\end{array}\right.
$$

where the sequences $\left\{w_{n}\right\},\left\{y_{n}\right\},\left\{\tilde{w}_{n}\right\},\left\{\tilde{y}_{n}\right\}$ are given by

$$
\left\{\begin{aligned}
&\left|w_{n+1}-w_{n}\right| \leq\left(1+\frac{1}{n+1}\right) \mathcal{H a u s}\left(T\left(u_{n+1}\right), T\left(u_{n}\right)\right) \quad \text { with } w_{n} \in T\left(u_{n}\right) \\
&\left|y_{n+1}-y_{n}\right| \leq\left(1+\frac{1}{n+1}\right) \mathcal{H a u s}\left(V\left(u_{n+1}\right), V\left(u_{n}\right) \quad \text { with } y_{n} \in V\left(u_{n}\right)\right. \\
&\left|\tilde{w}_{n+1}-\tilde{w}_{n}\right| \leq\left(1+\frac{1}{n+1}\right) \mathcal{H a u s}\left(T\left(z_{n+1}\right), T\left(z_{n}\right)\right) \quad \text { with } \tilde{w}_{n} \in T\left(z_{n}\right) \\
&\left|\tilde{y}_{n+1}-\tilde{y}_{n}\right| \leq\left(1+\frac{1}{n+1}\right) \mathcal{H a u s}\left(V\left(z_{n+1}\right), V\left(z_{n}\right)\right) \text { with } \tilde{y}_{n} \in V\left(z_{n}\right)
\end{aligned}\right.
$$


and the sequence of real numbers $\left\{\alpha_{n}, \beta_{n}\right\}$ satisfies

$$
0 \leq \alpha_{n}, \beta_{n} \leq 1 \text { for all } n \geq 0 \text { and } \sum_{n=0}^{+\infty} \alpha_{n}=+\infty \text {. }
$$

This iterative scheme is similar to the Ishikawa algorithm for solving quasi-variational inclusions and for suitable and appropriate choices of the involved operators, one can recover a number of algorithms for solving varaiational inclusions and related problems.

Theorem: Let the operator $N$ be strongly monotone with respect to $T$ with constant $\alpha>0$ and Lipschitz continuous with constants $\beta>0$ and $\eta>0$ with respect to the first and the second arguments respectively. Let $g$ be strongly monotone with constant $\sigma>0$ and Lipschitz continuous with constant $\delta>0$. Assume that $T$ and $V$ are Lipschitz continuous with constants $\xi>0$ and $\mu>0$ respectively. If the assumption (2.2) holds true and if we choose any $\varepsilon>0$ such that

$$
\begin{gathered}
\left|\lambda-\frac{\alpha-(1-k) \eta \xi(1+\varepsilon)}{\left(\beta^{2} \mu^{2}-\eta^{2} \xi^{2}\right)(1+\varepsilon)^{2}}\right|<\frac{\sqrt{(\alpha-(1-k) \eta \xi(1+\varepsilon))^{2}-k(1+\varepsilon)^{2}\left(\beta^{2} \mu^{2}-\eta^{2} \xi^{2}\right)(2-k)}}{\left(\beta^{2} \mu^{2}-\eta^{2} \xi^{2}\right)(1+\varepsilon)^{2}} \\
\alpha>\left((1-k) \eta \xi+(1+\varepsilon) \sqrt{k\left(\beta^{2} \mu^{2}-\eta^{2} \xi^{2}\right)(2-k)}\right) \\
\lambda \eta \xi(1+\varepsilon)<1-k \text { and } k=\nu+2 \sqrt{1-2 \sigma+\delta^{2}}
\end{gathered}
$$

then, there exist $u \in \mathcal{H}, w \in T(u), y \in V(u)$ satisfying the multivalued variational inclusion (2.1). Moreover, the sequences $\left\{u_{n}\right\},\left\{w_{n}\right\},\left\{y_{n}\right\},\left\{z_{n}\right\},\left\{\tilde{w}_{n}\right\},\left\{\tilde{y}_{n}\right\}$ generated by the algorithm (2.4) converge strongly to $u, w, y, u, \tilde{w}, \tilde{y}$ respectively.

Proof: Let $\mathcal{F}: u \rightarrow(1-t) u+t\left(u-g(u)+J_{\lambda}^{A_{u}}(g(u)-\lambda N(w, y))\right.$, where $w \in T(u), y \in V(u)$ and $0 \leq t \leq 1$. If the assumption (2.2) and hypothesis of the theorem are satisfied, then the same proof as the one we will give for proving the convergence of the algorithm shows that $\mathcal{F}$ is a contraction. So, by Banach principle, it follows that $\mathcal{F}$ has a unique fixed point, from which we deduce easily the existence of a solution $u$ with $w \in T(u)$ and $y \in V(u)$ verifying (2.1).

Now, let $u \in \mathcal{H}$ be a solution of (2.1). Then, we can write

$$
u=\left(1-\alpha_{n}\right) u+\alpha_{n}\left(u-g(u)+J_{\lambda}^{A_{u}}(g(u)-\lambda N(w, y)),\right.
$$

and

$$
u=\left(1-\beta_{n}\right) u+\beta_{n}\left(u-g(u)+J_{\lambda}^{A_{u}}(g(u)-\lambda N(w, y)) .\right.
$$

From (2.4), (2.9) and the convexity of the norm, we have

$$
\begin{aligned}
\left|u_{n+1}-u\right| & \leq\left(1-\alpha_{n}\right)\left|u_{n}-u\right|+\alpha_{n}\left|z_{n}-u-\left(g\left(z_{n}\right)-g(u)\right)\right| \\
& +\alpha_{n}\left|J_{\lambda}^{A_{z_{n}}}\left(g\left(z_{n}\right)-\lambda N\left(\tilde{w}_{n}, \tilde{y}_{n}\right)\right)-J_{\lambda}^{A_{u}}(g(u)-\lambda N(w, y))\right| \\
& \leq\left(1-\alpha_{n}\right)\left|u_{n}-u\right|+\alpha_{n}\left|z_{n}-u-\left(g\left(z_{n}\right)-g(u)\right)\right|
\end{aligned}
$$




$$
\begin{aligned}
& +\alpha_{n}\left|J_{\lambda}^{A_{z_{n}}}\left(g\left(z_{n}\right)-\lambda N\left(\tilde{w}_{n}, \tilde{y}_{n}\right)\right)-J_{\lambda}^{A_{z_{n}}}(g(u)-\lambda N(w, y))\right| \\
& +\alpha_{n}\left|J_{\lambda}^{A_{z_{n}}}(g(u)-\lambda N(w, y))-J_{\lambda}^{A_{u}}(g(u)-\lambda N(w, y))\right| \\
& \leq\left(1-\alpha_{n}\right)\left|u_{n}-u\right|+2 \alpha_{n}\left|z_{n}-u-\left(g\left(z_{n}\right)-g(u)\right)\right| \\
& +\alpha_{n}\left|z_{n}-u-\lambda\left(N\left(\tilde{w}_{n}, \tilde{y}_{n}\right)-N(w, y)\right)\right| \\
& \left.+\alpha_{n} \lambda \mid\left(N\left(w, \tilde{y}_{n}\right)\right)-N(w, y)\right)\left|+\alpha_{n} \nu\right| z_{n}-u \mid .
\end{aligned}
$$

On the other hand, a simple calculation involving the fact that $g$ is strongly monotone and Lipschitz continuous yields

$$
\left|z_{n}-u-\left(g\left(z_{n}\right)-g(u)\right)\right| \leq \sqrt{1-2 \sigma+\delta^{2}}\left|z_{n}-u\right| .
$$

Similarly, as $N$ is strongly monotone and Lipschitz continuous with respect to the first variable and thanks to Nadler's lemma, we obtain

$$
\begin{aligned}
\left|z_{n}-u-\lambda\left(N_{\tilde{y}_{n}}\left(\tilde{w}_{n}\right)-N_{\tilde{y}_{n}}(w)\right)\right|^{2} & =\left|z_{n}-u\right|^{2}-2 \lambda\left\langle N_{\tilde{y_{n}}}\left(\tilde{w}_{n}\right)-N_{\tilde{y}_{n}}(w), z_{n}-u\right\rangle \\
& +\lambda^{2}(1+\varepsilon)^{2} \mathcal{H} \operatorname{aus}^{2}\left(N_{\tilde{y}_{n}}\left(\tilde{w}_{n}\right), N_{\tilde{y}_{n}}(w)\right) \\
& \leq\left(1-2 \lambda \alpha+(1+\varepsilon)^{2} \lambda^{2} \beta^{2} \mu^{2}\right)\left\|z_{n}-u\right\|^{2} .
\end{aligned}
$$

From which we infer

$$
\mid z_{n}-u-\lambda\left(N_{\tilde{y}_{n}}\left(\tilde{w}_{n}\right)-N_{\tilde{y}_{n}}(w)\left|\leq \sqrt{1-2 \lambda \alpha+(1+\varepsilon)^{2} \lambda^{2} \beta^{2} \mu^{2}}\right| z_{n}-u \mid .\right.
$$

Now, the Lipschitz continuity of $N$ with respect to the second argument together with the Lipschitz continuity of $V$ gives

$$
\begin{aligned}
\left|N\left(w, \tilde{y}_{n}\right)-N(w, y)\right| & \leq \eta(1+\varepsilon)\left|\tilde{y}_{n}-y\right| \\
& \leq \eta(1+\varepsilon) \mathcal{H a u s}\left(V\left(z_{n}\right), V(u)\right) \\
& \leq \eta \xi(1+\varepsilon)\left|z_{n}-u\right| .
\end{aligned}
$$

Combining the latter inequality with (2.11) and (2.13), we obtain

$$
\begin{aligned}
\left|u_{n+1}-u\right| & \leq\left(1-\alpha_{n}\right)\left|u_{n}-u\right|+\alpha_{n}(k+\lambda \eta \xi(1+\varepsilon)+t(\lambda))\left|z_{n}-u\right| \\
& \leq\left(1-\alpha_{n}\right)\left|u_{n}-u\right|+\alpha_{n} \theta\left|z_{n}-u\right|,
\end{aligned}
$$

where $k=\nu+2 \sqrt{1-2 \sigma+\delta^{2}}, \theta=k+\lambda \eta \xi(1+\varepsilon)+t(\lambda)$ and $t(\lambda)=\sqrt{1-2 \lambda \alpha+\lambda^{2}(1+\varepsilon)^{2} \beta^{2} \mu^{2}}$. In similar way, in the light of (2.4) and (2.10), we infer

$$
\begin{aligned}
\left|z_{n}-u\right| & \leq\left(1-\beta_{n}\right)\left|u_{n}-u\right|+2 \beta_{n}\left|u_{n}-u-\left(g\left(u_{n}\right)-g(u)\right)\right| \\
& \left.+\beta_{n} \mid u_{n}-u-\lambda\left(N\left(w_{n}, y_{n}\right)\right)-N(w, y)\right) \mid \\
& \leq\left(1-\beta_{n}\right)\left|u_{n}-u\right|+\beta_{n} \theta\left|u_{n}-u\right| \\
& \leq\left|u_{n}-u\right| \text { because } 0 \leq \theta<1 .
\end{aligned}
$$


Taking into account the latter inequality, we finally obtain

$$
\begin{aligned}
\left|u_{n+1}-u\right| & \leq\left(\left(1-\alpha_{n}(1-\theta)\right)\left|u_{n}-u\right|\right. \\
& \leq \prod_{i=0}^{n}\left(\left(1-\alpha_{i}(1-\theta)\right)\left|u_{0}-u\right| .\right.
\end{aligned}
$$

From conditions on the parameters, it follows that $\theta<1$ and since $\sum_{n=0}^{\infty} \alpha_{n}$ diverges and $1>\theta \geq 0$, we have $\sum_{i=0}^{\infty}\left\{1-(1-\theta) \alpha_{i}\right\}=0$. Hence the sequence $\left\{u_{n}\right\}$ converges strongly to $u$. Also from (2.14), we see that the sequence $\left\{z_{n}\right\}$ converges also to $u$. One can easily show that the sequences $\left\{w_{n}\right\},\left\{y_{n}\right\},\left\{\tilde{w}_{n}\right\},\left\{\tilde{y}_{n}\right\}$ are Cauchy sequences and thus converge strongly to some $w, y, \tilde{w}, \tilde{y}$ respectively. Indeed, for $\left\{w_{n}\right\}$, we can write

$$
\begin{aligned}
\left|w_{n+1}-w_{n}\right| & \leq\left(1+\frac{1}{n+1}\right) \mathcal{H a u s}\left(T\left(u_{n+1}\right), T\left(u_{n}\right)\right) \\
& \leq\left(1+\frac{1}{n+1}\right) \xi\left|u_{n+1}-u_{n}\right|
\end{aligned}
$$

In a similar way, one shows that $\left\{y_{n}\right\},\left\{\tilde{w}_{n}\right\},\left\{\tilde{y}_{n}\right\}$ are also Cauchy sequences.

We will now show that $w \in T(u), y \in V(u), \tilde{w} \in T(x), \tilde{y} \in V(x)$. In fact, for $\left\{w_{n}\right\}$, we have

$$
\begin{aligned}
\operatorname{dist}(w, T(u)) & \leq\left|w-w_{n}\right|+\operatorname{dist}\left(w_{n}, T(u)\right) \\
& \leq\left|w-w_{n}\right|+\mathcal{H a u s}\left(T\left(u_{n}\right), T(u)\right) \\
& \leq\left|w-w_{n}\right|+\xi\left|u_{n}-u\right| \rightarrow 0 \text { as } n \rightarrow+\infty
\end{aligned}
$$

where $\operatorname{dist}(w, T(u))=\inf \{|w-z|: z \in T(u)\}$. From which, we deduce $\operatorname{dist}(w, T(u))=0$. This implies that $w \in T(u)$, because $T(u)$ is closed. In a similar way, one shows that $y \in V(u), \tilde{w} \in T(u), \tilde{y} \in V(u)$.

Finally, by noting that the relation

$$
\left|J_{\lambda}^{A_{u_{n}}}(w)-J_{\lambda}^{A_{u}}(w)\right| \leq \nu\left|u_{n}-u\right|
$$

implies that $\lim _{n \rightarrow+\infty}\left|J_{\lambda}^{A_{u_{n}}} w-J_{\lambda}^{A_{u}} w\right|=0$, by taking into account the Lipschitz continuity of the involved operators and by passing to the limit in the first relation of (2.4), we obtain

$$
g(u)=J_{\lambda}^{A_{u}}(g(u)-\lambda N(w, y)),
$$

which amounts to saying that $u$ is a solution of (2.1). This completes the proof.

In the next proposition, we will identify operators with their graphs and consider the bounded Hausdorff distance between $A_{u}$ and $A_{v}$ which is defined by:

$$
\mathcal{H} a u s_{\rho}\left(A_{u}, A_{v}\right):=\max \left\{\mathrm{e}\left(A_{u} \cap \rho \mathcal{B}_{\mathcal{H} \times \mathcal{H}}, A_{v}\right), \mathrm{e}\left(A_{v} \cap \rho \mathcal{B}_{\mathcal{H} \times \mathcal{H}}, A_{u}\right)\right\}
$$

where $\rho \mathcal{B}_{\mathcal{H} \times \mathcal{H}}$ stands for the closed ball of $\mathcal{H} \times \mathcal{H}$ with radius $\rho$ and centred at the origin.

Proposition: If the following condition holds true

$$
\mathcal{H} a u s_{\rho}\left(A_{u}, A_{v}\right) \leq \tau|u-v|, \quad \forall u, v \in \mathcal{H} \forall \rho \geq 0,
$$




$$
\left|J_{\lambda}^{A_{u}}(w)-J_{\lambda}^{A_{v}}(w)\right| \leq \nu|u-v|,
$$

with $\nu=(2+\lambda) \tau$.

Proof: According to ([2], proposition 1.2), we can write

$$
\left|J_{\lambda}^{A_{u}}(w)-J_{\lambda}^{A_{v}}(w)\right| \leq(2+\lambda) \mathcal{H} a u s_{\rho}\left(A_{u}, A_{v}\right),
$$

with $\rho=\max \left\{|w|+\left|J_{\lambda}^{A_{u}}(0)\right|, \frac{1}{\lambda}\left(|w|+\left|J_{\lambda}^{A_{u}}(0)\right|\right)\right.$.

This combined with the hypothesis gives the desired result.

Furthermore, in the case when $A_{u}$ is the partial differential of the indicator function of a closed convex set $K(u)$, we can replace condition (2.16) by a condition in terms of $\{K(u)\}$, namely

$$
\mathcal{H} \operatorname{aus}_{\rho}^{\frac{1}{2}}(K(u), K(v)) \leq \tau|u-v|, \quad \forall u, v \in \mathcal{H} \forall \rho \geq 0 .
$$

Indeed, ([2], remark 2.5) ensures the existence of two constants $K_{\rho}$ and $\rho$ ' which depend on $\rho$ such that

$$
\mathcal{H a u s} s_{\rho}\left(\partial \delta_{K(u)}, \partial \delta_{K(v)}\right) \leq K_{\rho} \mathcal{H} \operatorname{aus}_{\rho^{\prime}}^{\frac{1}{2}}(K(u), K(v)) .
$$

\section{Remark:}

(i) It is worth mentioning that the condition (2.2) implies the convergence of the resolvents $\left(J_{\lambda}^{A_{u_{n}}}\right)$ which is equivalent to the graph convergence of the operators $\left(A_{u_{n}}\right)$. While condition (2.16) implies the convergence of $\left(A_{u_{n}}\right)$ with respect to the bounded Hausdorff distance which is equivalent to the convergence of the resolvents on bounded sets and which is stronger than the graph convergence of the operators $\left(A_{u_{n}}\right)$ (see, for example, [2]).

(ii) For clarity's sake we worked in a Hilbert space setting, but our results are still valid in reflexive Banach space.

\section{References}

1. W. F. Ames, Numerical Methods for Partial Differential Equations, Third Edition, Academic Press, New York, 1992.

2. H. Attouch, H. Riahi and A. Moudafi Quantitative stability analysis for maximal monotone operators and semi-groups of contractions, J. Nonlinear Anal., Theo. Meth. \& Appli., 21, (1993), 697-723.

3. C. Baiocchi and A. Capelo, Variational and Quasi-Variational Inequalities, J. Wiley and Sons, New York, London, 1984.

4. H. Brézis. Opérateurs Maximaux Monotone et Semigroups de Contractions dans les Espaces de Hilbert, North-Holland, Amsterdam, 1973. 
5. S. S. Chang, J. K. Kim and K. H. Kim, On the existence and iterative approximation problems of solutions for set-valued variational inclusions in banach space. To appear in J. Math. Anal. Appli.

6. R.W. Cottle, F. Giannessi and J.L. Lions, Variational Inequalities and Complementarity Problems: Theory and Applications, J. Wiley and Sons, New York, 1980.

7. F. Giannessi and A. Maugeri, Variational Inequalities and Network Equilibrium Problems, Plenum Press, New York. 1995.

8. R. Glowinski, J.L. Lions and R. Trémolières, Numerical Analysis of Variational Inequalities, North-Holland, Amsterdam, 1981.

9. R. Glowinski, Numerical Methods for Nonlinear Variational Problems, Springer-Verlag, Berlin, 1984.

10. R. Glowinski and P. Le Tallec, Augmented Lagrangian and Operator-Splitting Methods in Nonlinear Mechanics, SIAM Publication Co. Philadelphia, 1989.

11. B. He, A class of projection and contraction methods for monotone variational inequalities, Appl. Math. Optim, 35, (1997), 69-76.

12. B. Martinet, Regularization d'inequations variationelles par approximations successives, Rev. Francaise d'Auto. et Inform. Rech. Oper., 4, (1972), 154-159.

13. A. Moudafi and M. Aslam Noor, Sensitivity analysis for variational inclusions by the Wiener-Hopf equations techniques, J. Appl. Math. Stochastic Anal., 12, (1999), 223232.

14. A. Moudafi and M. Aslam Noor, New convergence results for iterative methods for set-valued mixed variational inequalities, J. Math. Ineq. Appli., 3, (2000), 223-232.

15. S. B. Nadler, Multivalued contraction mappings, Pacific J. Math., 30, (1969), 475-488.

16. M. Aslam Noor, Algorithms for general monotone mixed variational inequalities, J. Math. Anal. Appl., 229, (1999),295-303.

17. M. Aslam Noor. Projection-splitting algorithms for monotone variational inequalities, Computer Math. Applic., 39, (2000), 73-79.

18. M. Aslam Noor, General monotone mixed variational inequalities, J. Natural Geometry, 17, (2000),59-76.

19. G. B. Passty, Ergodic convergence to a zero of the sum of maximal monotone operators, Jour. Math. Anal. Appl., 7, (1978), 591-597.

20. S.M. Robinson, Generalized equations and their solutions, Math. Program. Studies, 10, (1979), 128-141.

21. S. M. Robinson, Normal maps induced by linear transformations, Math. Oper. Research, 17, (1992),691-714.

22. R.T. Rockafellar, Monotone operator and the proximal point algorithm, SIAM J. Control. Opt., 14 (5), (1976), 877-898. 
23. P. Shi, Equivalence of variational inequalities with Wiener-Hopf equations, Proc. Amer. Math. Soc., 111, (1991), 339-346.

24. P. Tseng, A modified forward-backward splitting method for maximal monotone mappings, SIAM J. Control Optim., 38, (2000), 431-446.

(*) Abdellatif Moudafi, Univeristé Antilles Guyane, GRIMAAG, département scientifique interfacultaire, 97200 Schoelcher, Martinique, France. abdellatif.moudafi@martinique.univ-ag.fr

(**) Muhammad Aslam Noor, Mathematics, Etisalat College of Engineering, P. O. Box 980, Sharjah, United Arab Emirates. noor@ece.ac.ae 